\title{
Modelling of a Two-signal SFQ Detection Scheme for the Readout of Superconducting Nanowire Single Photon Detectors
}

\author{
Connor D. Shelly, Patrick See, \\ Jane Ireland and Jonathan M. Williams London Centre of Nanotechnology \\ National Physical Laboratory \\ Teddington, TW11 0LW, UK \\ Email: connor.shelly@npl.co.uk
}

\author{
Alessandro Casaburi \\ and Robert H. Hadfield \\ School of Engineering, Glasgow University \\ Rankine Building, Oakfield Avenue, \\ Glasgow, G12 8LT, UK
}

\begin{abstract}
We present a two-signal single flux quantum (SFQ) detection scheme for the purpose of reading out two pixels of a superconducting nanowire single photon detector (SNSPD) [1]. The circuit is based on a coincidence buffer element which is able to output a signal when both of its input lines are triggered [2]. The circuit model for the SNSPD element is simulated in SPICE and optimized to match the experimental SNSPD response data. The two-signal detection scheme is simulated using JSIM which allows for the simulation of Josephson junction elements in a circuit. We demonstrate a model of the two-signal circuit operating with two simulated SNSPD pixel inputs and investigate the response of the scheme when a phase shift is applied to one of the inputs. The scheme shows potential as a useful coincidence detector of single photons. We also present preliminary experimental results of nanobridge-based Josephson junctions to be used in the realization of the coincidence detector circuit. Evidence of the nanobridges exhibiting Josephson behavior (SQUID modulation) are presented.
\end{abstract}

Index Terms-Single Flux Quantum (SFQ) logic, detector readout, superconducting nanowire.

\section{INTRODUCTION}

Superconducting Nanowire Single Photon Detectors (SNSPDs) are a key quantum technology allowing single photon detection at infrared wavelengths. In addition, a number of application areas exist including quantum key distribution (QKD), time-of-flight ranging and quantum enhanced imaging [1]. A major barrier to SNSPD many-pixel array technology is a suitable scalable readout technology. Current readout schemes proposed from leading groups in the US (NIST, JPL and MIT) aimed at scaling up the area (number of pixels) of the SNSPD arrays are hampered by the difficulties in achieving high readout rates and by the high thermal load coming from a large number of bias and readout lines [3], [4], [5]. It is therefore advantageous to perform signal processing at cryogenic temperatures in order to minimize heat load on the cooling system. Recently, a promising readout scheme based on the RF coupling of the SNSPDs with coplanar waveguide have been shown by the

This work is funded as part of two feasibility studies funded by Innovate UK: Project numbers 131874 and 102677. Co-funding supported by the UK NMS Electromagnetics and Time Programme and the EPSRC. technology is still at an early stage [6]. One possible readout technology is that of Single Flux Quantum (SFQ) circuitry [2], in this case using superconducting nano-bridge Josephson junctions. SFQ readout has successfully been employed as a readout technique using traditional tunnel junctions [7]. An alternative fabrication technology is employed in the work here through use of nanobridge weak link Josephson junctions [8]. The main advantage of the nanobridge technology that we present is that it is a single-layer process defined using electron-beam lithography with no need for multi-layer fabrication or highly controlled oxide layers. This makes nanobridges made in this way a very promising route for scaling up to large numbers of junctions which is required for any multi-pixel readout scheme. A secondary advantage for single-layer nanobridge technology is that the fabrication can be more easily integrated with that of the SNSPD devices which are typically made out of thin-film superconducting niobium-nitride $(\mathrm{NbN})$ and made using e-beam lithography. For the proof-of-principle devices presented in this work we use pure niobium which has the advantage that the coherence length is greater than that of $\mathrm{NbN}$ which allows our devices to exhibit the Josephson effect in larger dimension nanobridges.

\section{Fabrication And Measurement of Weak Link NANOBRIDGES}

The nanobridge devices are fabricated using a combination of electron beam lithograpy (EBL) and dry etching using an aluminium hard mask. A $150 \mathrm{~nm}$ thick layer of niobium is sputtered onto a silicon substrate. PMMA resist is used for the EBL patterning of the nanobridge. A hard mask using $70 \mathrm{~nm}$ of thermally deposited aluminium is then created. The $\mathrm{Nb}$ is dry etched into the $\mathrm{Si}$ substrate using a $\mathrm{CHF}_{3} / \mathrm{SF}_{6}$ plasma. Finally, the $\mathrm{Al}$ hard mask is removed using a standard photoresist developer. A scanning electron micrograph of an example nanobridge SQUID is shown in Fig. 1.

Electrical measurements of the nano-bridge weak links are carried out using a dip probe in a helium dewar $(4.2 \mathrm{~K})$. The temperature is varied by raising and lowering the probe and the temperature is monitored using a silicon diode thermometer 


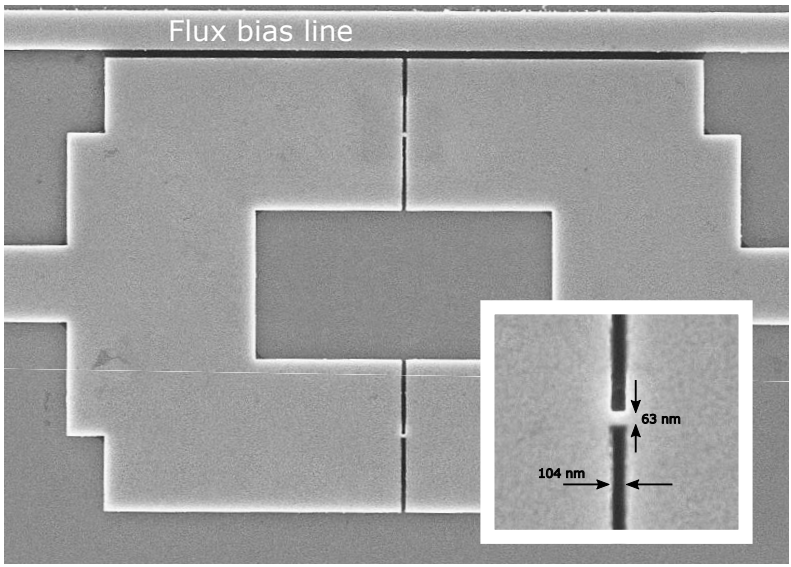

Fig. 1. Scanning electron micrograph of a niobium nanobridge SQUID showing the flux bias line at the top of the image. The inset shows a single nanobridge at greater magnification to illustrate the dimensions. The designed dimensions of the bridges are $60 \mathrm{~nm} \times 100 \mathrm{~nm}$.

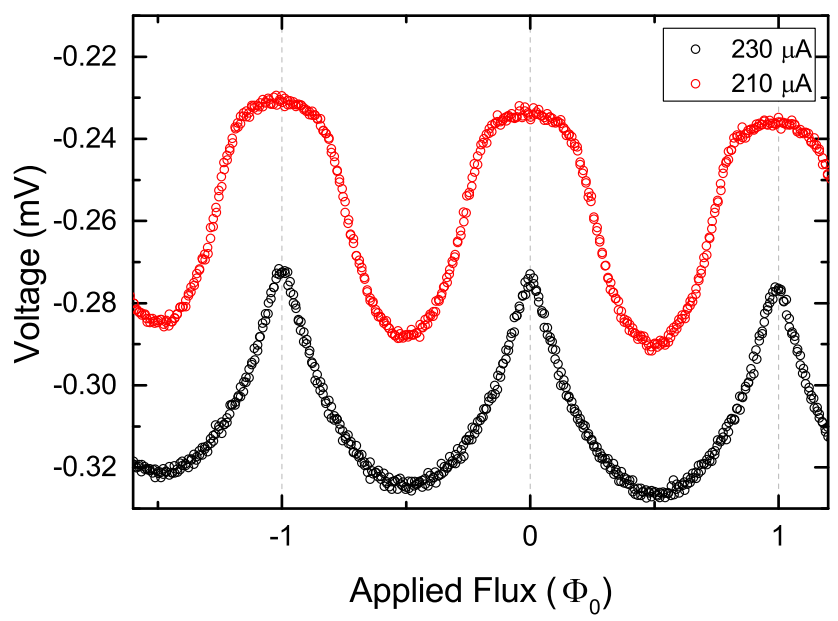

Fig. 2. $V-\Phi$ modulation at two dc bias currents of the nanobridge SQUID. Applied flux is varied by passing a current through a superconducting line placed $500 \mathrm{~nm}$ away from the SQUID loop. By applying different dc bias currents $(210 \mu \mathrm{A}$ and $230 \mu \mathrm{A})$ to the SQUID, the modulation characteristic is changed.

mounted to the same copper plate as the sample. For the nanobridges to function as SFQ elements it is necessary that the weak links exhibit Josephson junction behaviour. To this end, we demonstrate voltage-flux $(V-\Phi)$ modulation of a SQUID device containing two $60 \times 100 \mathrm{~nm}$ bridges. A flux bias line placed $500 \mathrm{~nm}$ away from the SQUID loop is used to modulate the flux which results in the characteristic $V-\Phi$ modulation as shown in Fig. 2. We have also observed the characteristic frequency-dependent voltage steps (Shapiro steps) as a result of coupling of a microwave current into a single nanobridge (not shown) [9]. These two properties demonstrate that our nanobridge devices exhibit the Josephson effect and can be used as elements in more complex SFQ circuits.

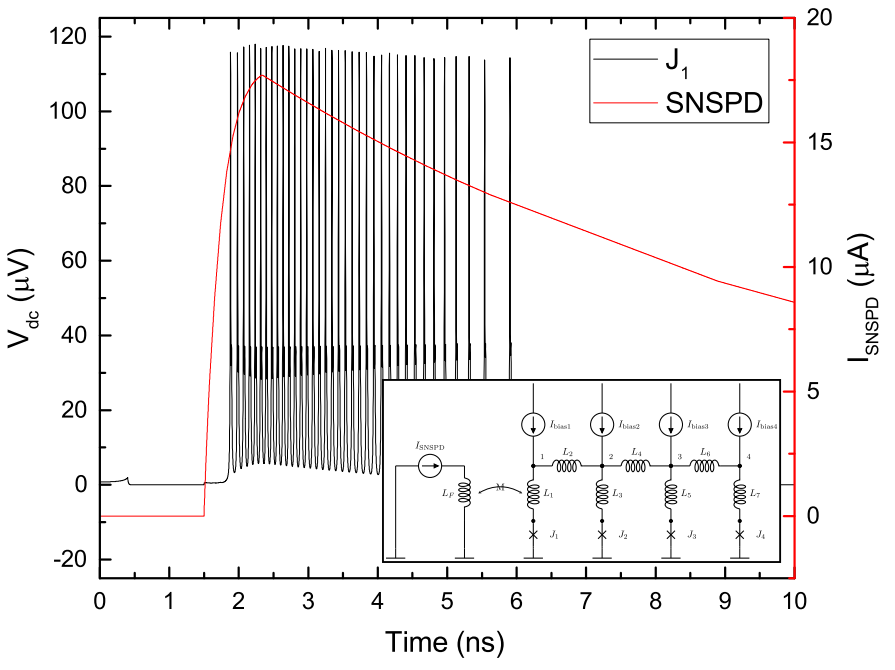

Fig. 3. Simulated response of the SNSPD to an incident photon (red line). The SFQ circuit (inset) responds with a number of SFQ pulses during the dead time of the SNSPD.

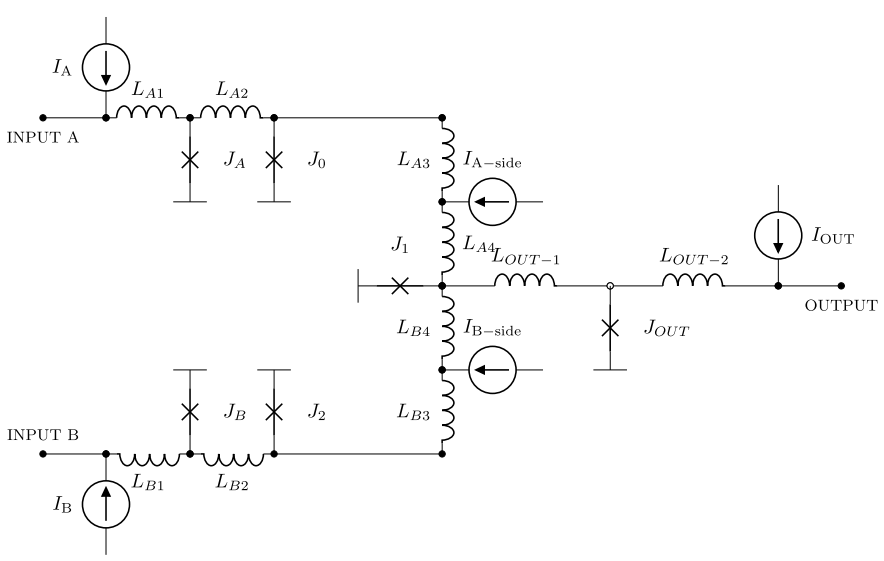

Fig. 4. Circuit diagram of the Coincidence Buffer as simulated in JSIM. The values for elements are shown in Table I. Inputs A and B represent input pulses that will be taken from two independent SNSPDs.

\section{Modelling of the Two-PiXel Coincidence CIRCUIT}

The circuit simulations are performed using JSIM, a circuit simulator optimised for solving superconducting circuits including non-linear Josephson junction elements [10]. Simple Josephson Transmission Line (JTL) circuits inductively coupled to the SNSPD response are simulated in JSIM. We observe that with sufficient current bias to the Josephson junctions of the JTL circuit many SFQ pulses can occur in the 'dead time' $(\approx 10 \mathrm{~ns})$ of the SNSPD as shown in Fig. 3 .

As the most simple scenario we have chosen to implement a two-pixel coincidence detection circuit based on the 'coincidence buffer' taken from the Stony Brook RSFQ Cell Library (more details found in [2] and [11]) as shown in Fig. 4. The element consists of two loops: $J_{0}-L_{3}-J_{1}$ and $J_{2}-L_{4}-J_{1}$. Each loop can hold an incoming SFQ pulse from either of the inputs A or B. When both A and B arrive (in any order, 
TABLE I

PARAMETERS USED FOR JSIM MODELLING. ALL BIAS CURRENTS IN THE CIRCUIT ARE SET TO $93 \mu \mathrm{A}$

\begin{tabular}{c|c} 
Inductors & Junction $I_{\mathrm{C}}$ \\
\hline$L_{A 1}=L_{B 1}=2.66 \mathrm{pH}$ & $J_{A}=J_{B}=100 \mu \mathrm{A}$ \\
$L_{A 2}=L_{B 3}=4.11 \mathrm{pH}$ & $J_{0}=J_{2}=139 \mu \mathrm{A}$ \\
$L_{A 3}=L_{B 3}=1.78 \mathrm{pH}$ & $J_{1}=188 \mu \mathrm{A}$ \\
$L_{A 4}=L_{B 4}=4.11 \mathrm{pH}$ & \\
$L_{\text {OUT-1 }}=3.75 \mathrm{pH}$ & \\
$L_{\text {OUT-2 }}=2.90 \mathrm{pH}$ &
\end{tabular}

and with any delay) they result in a switch of junction $J_{1}$ producing an output.

Although the coincidence buffer is not sensitive to the timing of the input pulses, we operate the SFQ circuit in the regime that one SNSPD pulse results in many SFQ pulses. This means that the average voltage which is measured would only be above the measurement threshold during times that both SNSPD pulses are overlapping in time. This allows operation of a time-sensitive single-photon coincidence detector.

Fig. 5 schematically demonstrates how the SFQ circuit would be used as a coincidence detector of single photons. The red and blue hatched boxes at the top of the figure represent the incoming input pulses at port $\mathrm{A}$ and port $\mathrm{B}$ as a result of incident photons at the SNSPD. When there is no overlap of the incoming photons, no SFQ pulses are produced in the circuit and no voltage at the circuit output will be measured. If the incident photons are overlapping in time-of-arrival then the SFQ circuit will produce pulses that result in a measured average voltage at the circuit output. Maximal output voltage is achieved when the incident photons have $100 \%$ overlap.

\section{CONCLUSION}

In conclusion we demonstrate successful fabrication of superconducting nanobridges made from niobium down to sub-50 nm dimensions. Low-temperature measurements (sub$9 \mathrm{~K})$ were performed with the SQUID devices exhibiting characteristic Josephson behaviour of $V-\Phi$ modulation. Additional measurements of single nanobridges demonstrate further evidence of Josephson behaviour with characteristic frequency-dependent voltage steps when subject to microwave radiation [9]. We also model an SFQ circuit known as a 'coincidence buffer' in Josephson circuit simulator JSIM and propose its potential use as a time-resolving two-photon coincidence detector. Our modelling demonstrates that the circuit can be used by measuring the average voltage across the device output and that the amplitude of this average voltage can give information on the temporal overlap of the incident photons.

\section{REFERENCES}

[1] C. M. Natarajan, M. G. Tanner, and R. H. Hadfield, "Superconducting nanowire single-photon detectors: physics and applications," Superconductor Science and Technology, vol. 25, no. 6, p. 063001, 2012.

[2] K. Likharev and V. Semenov, "RSFQ logic/memory family: a new Josephson-junction technology for sub-terahertz-clock-frequency digital systems," IEEE Transactions on Appiled Superconductivity, vol. 1, p. 13, 1991.

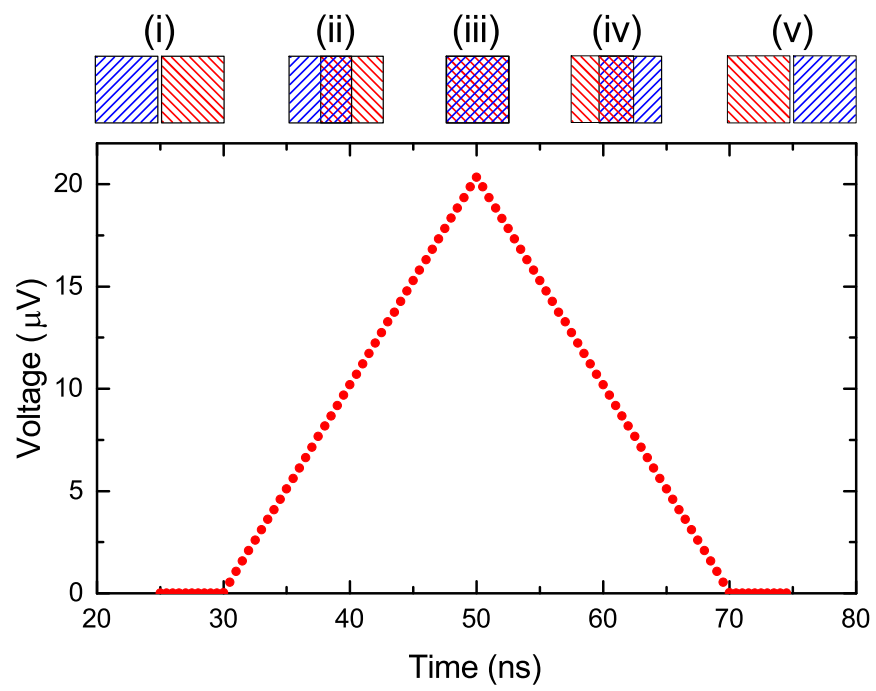

Fig. 5. Simulated voltage measured across junction $J_{1}$. The schematic at the top of the figure shows the two input pulses at port $A$ (blue) and port $B$ (red). The red pulse timing is kept constant throughout the measurement whilst the blue pulse has a variable delay. At the beginning (i) the blue pulse occurs before the red pulse with no overlap. This results in no SFQ pulses at $J_{1}$. As the blue pulse timing is varied so that there is some overlap (ii), some SFQ pulses are produced due to the signal coincidence. The peak number of pulses and thus the peak voltage measured occurs when there is $100 \%$ overlap (iii) between the input signals. As the blue pulse continues past the red pulse in time the output voltage is reduced again down to zero ((iv) and (v)).

[3] M. S. Allman, V. B. Verma, M. Stevens, T. Gerrits, R. D. Horansky, A. E. Lita, F. Marsili, A. Beyer, M. D. Shaw, D. Kumor, R. Mirin, and S. W. Nam, "A near-infrared 64-pixel superconducting nanowire single photon detector array with integrated multiplexed readout," Applied Physics Letters, vol. 106, no. 19, p. 192601, 2015.

[4] Q.-Y. Zhao, A. McCaughan, F. Bellei, F. Najafi, D. D. Fazio, A. Dane, Y. Ivry, and K. K. Berggren, "Superconducting-nanowire single-photondetector linear array," Applied Physics Letters, vol. 103, no. 14, p. 142602, 2013.

[5] Q.-Y. Zhao, D. Zhu, N. Calandri, A. E. Dane, A. N. McCaughan, F. Bellei, H.-Z. Wang, D. F. Santavicca, and K. K. Berggren, "Singlephoton imager based on a superconducting nanowire delay line," Nat Photon, vol. 11, no. 4, pp. 247-251, Apr. 2017.

[6] S. Doerner, A. Kuzmin, S. Wuensch, K. Ilin, and M. Siegel, "Operation of superconducting nanowire single-photon detectors embedded in lumped-element resonant circuits," IEEE Transactions on Applied Superconductivity, vol. 26, no. 3, pp. 1-5, April 2016.

[7] H. Terai, S. Miki, and Z. Wang, "Readout electronics using single-fluxquantum circuit technology for superconducting single-photon detector array," IEEE Transactions on Applied Superconductivity, vol. 19, no. 3, pp. 350-353, June 2009.

[8] K. K. Likharev, "Superconducting weak links," Rev. Mod. Phys., vol. 51, pp. 101-159, Jan 1979.

[9] C. D. Shelly, P. See, J. Ireland, E. J. Romans, and J. M. Williams, "Weak link nanobridges as single flux quantum elements," unpublished.

[10] E. S. Fang and T. V. Duzer, "A Josephson integrated circuit simulator (JSIM) for superconductive electronics application," in Extended $A b$ stracts of 1989 Intl. Superconductivity Electronics Conf. (ISEC'89), pp. 407-410., 1989.

[11] O. A. Mukhanov, S. V. Rylov, V. K. Semonov, and S. V. Vyshenskii, "RSFQ logic arithmetic," IEEE Transactions on Magnetics, vol. 25, no. 2, pp. 857-860, Mar 1989. 